This item was submitted to Loughborough's Research Repository by the author.

Items in Figshare are protected by copyright, with all rights reserved, unless otherwise indicated.

\title{
Maturational timing, physical self-perceptions and physical activity in UK adolescent females: investigation of a mediated effects model
}

PLEASE CITE THE PUBLISHED VERSION

https://doi.org/10.1080/03014460.2020.1784277

\section{PUBLISHER}

Informa UK Limited, trading as Taylor \& Francis Group

VERSION

AM (Accepted Manuscript)

\section{PUBLISHER STATEMENT}

This is an Accepted Manuscript of an article published by Taylor \& Francis in Annals of Human Biology on 30 September 2020, available online: http://www.tandfonline.com/10.1080/03014460.2020.1784277.

\section{LICENCE}

CC BY-NC-ND 4.0

\section{REPOSITORY RECORD}

Cumming, Sean P, Deirdre M Harrington, Melanie J Davis, Charlotte L Edwardson, Trish Gorely, Kamlesh Khunti, Alex V Rowlands, Thomas Yates, and Lauren Sherar. 2020. "Maturational Timing, Physical Selfperceptions and Physical Activity in UK Adolescent Females: Investigation of a Mediated Effects Model". Loughborough University. https://hdl.handle.net/2134/13240946.v1. 
MATURATION, PHYSICAL SELF, PHYSICAL ACTIVITY

11 Sean Cumming, Department for Health, University of Bath, Bath, BA2 7AY

12 Deirdre M. Harrington, Alex Rowlands, Melanie J. Davies, Kamlesh Khunti, Thomas

13 Yates \& Charlotte L. Edwardson. University of Leicester, Diabetes Research Centre,

14 Leicester General Hospital, Leicester, LE5 4PW, UK

15 Melanie J. Davies, Alex Rowlands, Thomas Yates \& Charlotte L. Edwardson

16 NIHR Leicester Biomedical Research Centre, Leicester, Leicester General Hospital,

17 Leicester, LE5 4PW, UK

18 Kamlesh Khunti, Applied Health Research and Care East Midlands, Leicester General

19 Hospital, Leicester, LE5 4PW, UK

20 Trish Gorely, Centre for Health Science, University of the Highlands and Islands,

21 Inverness, IV2 3JH, UK

22 Lauren B. Sherar, School of Sport, Exercise and Health Sciences, Loughborough

23 University, Loughborough, LE11 3TU, UK

25 Key Words: Adolescence, Exercise, Self-worth, Body attractiveness 
1 Abstract Word Count: 216

2 Manuscript Word Count: 3270 
Abstract

3 Background: Advanced (early) biological maturation may be a risk factor for inactivity

4 among adolescent girls. The aim of the present paper was to test the mediational effects

5 of body attractiveness and physical self-worth on the relationship between biological

6 maturity and accelerometer assessed moderate-to-vigorous physical activity (MVPA) in a

7 large multi-ethnic sample of girls from the Midlands area in the UK (11-14 years).

8 Methods: Biological maturity (predicting age at peak height velocity (APHV)); self-

9 perceptions of body attractiveness, physical self-worth, and minutes spent in MVPA were

10 assessed in 1062 females aged 11 to 14 years.

11 Results: Structural equation modeling using maximum likelihood estimation and boot-

12 strapping procedures supported the hypothesized model. Later maturation predicted

13 higher perceptions of body attractiveness $(\beta=.25, \mathrm{p}<.001)$ which, in turn, predicted higher

14 perceptions of physical self-worth $(\beta=.91, p<.001)$ and, significantly higher MVPA

$15(\beta=.22, \mathrm{p}<.001)$. Examination of the bootstrap-generated bias-corrected confidence

16 intervals suggested that perceptions of body attractiveness and physical self-worth

17 partially mediated a positive association between predicted APHV and MVPA $(\beta=.05$,

$18 \mathrm{p}<.001)$.

19 Conclusions: Greater biological maturity (i.e. early maturity) in adolescent girls is

20 associated with less involvement in MVPA and appears to be partly explained by lower

21 perceptions of body attractiveness and physical self-worth. Physical activity interventions

22 should consider girls perceptions of their pubertal related physiological changes during

23 adolescence, particularly among early maturing girls. 
MATURATION, PHYSICAL SELF, PHYSICAL ACTIVITY

\section{Maturational Timing, Physical Self-Perceptions and Physical Activity in UK} Adolescent Females: Investigation of a Mediated Effects Model

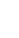

$$
\begin{aligned}
& \text { Introduction } \\
& \text { Maintaining at least the recommended level of moderate-to-vigorous physical activity } \\
& \text { (MVPA) is a well-accepted lifestyle target for promoting physical and mental health in } \\
& \text { adults as well as children and young people (Ekelund et al. } 2012) \text {. Evidence suggests, } \\
& \text { however, that a significant proportion of adolescents }(77 \cdot 6 \% \text { of boys and } 84 \cdot 7 \% \text { of girls) } \\
& \text { globally do not achieve this minimum recommended level (Guthold et al. } 2019 \text { Nov). } \\
& \text { Adolescence is recognized as a period of great physical, psychosocial, cognitive, }
\end{aligned}
$$$$
\text { and emotional change. Variance in the age that an individual enters into puberty may }
$$$$
\text { contribute towards physical (in)activity during adolescence, especially among girls }
$$

(Malina, Robert M. Bouchard, Claude. Bar-Or 2004). Biological maturation refers to the progress towards the mature state and can be defined in terms of status (specific stage of maturation), tempo (rate at which maturation occurs) and timing (age at which specific milestones, such as age at menarche or age at peak height velocity (APHV) occurs).

Children of the same chronological age can differ significantly in their degree of biological maturation with some individuals maturing earlier or later than their peers (Malina, Robert M. Bouchard, Claude. Bar-Or 2004). Maturation has been proposed as one reason why girls tend to be less active (Sherar et al. 2007) and more sedentary (Machado Rodrigues et al. 2010) than boys of the same chronological age. Consistent with this finding, girls who are advanced in maturation for their age (i.e. early maturers) have also been shown to be less active 
1 (Cumming, Gillison, et al. 2011) and more sedentary (Machado Rodrigues et al. 2010)

2 than later maturing girls of the same age (i.e. late maturers). This effect may be greater

3 when considered at the extreme ends of the maturity continuum (i.e., most versus least

4 mature girls) (Cumming, Sherar, Smart, et al. 2012). Evidence supporting the association

5 between maturation and physical activity is, however equivocal, potentially reflecting

6 variation in the definitions and measures of both biological maturity and physical activity,

7 small sample sizes, and a general failure to consider potential mediating and/or

8 moderating factors (Sherar et al. 2010).

9 The relationship between maturation and physical activity in girls is complex and

10 multifaceted. The Biocultural Model of Maturity Associated Variance in Physical

11 Activity (Cumming, Sherar, Pindus, et al. 2012) posits that biological maturation may

12 directly and/or indirectly impact activity in youth. Specifically, it argues that the impact

13 of maturation on physical activity may be mediated and/or moderated by endogenous

14 (e.g., beliefs, ideas, self-perceptions) or exogenous (e.g., societal and cultural

15 expectations and values, environment) factors, respectively. That is, perceptions of

16 physical changes during puberty and the reactions and evaluations of others may be as, if

17 not more, important than the changes themselves. In support of these contentions,

18 perceptions of the physical-self, in particular attractiveness and sports competence has

19 been shown to mediate inverse relations between biological maturation and self-reported

20 physical activity in a sample $(\mathrm{n}=244)$ of $11-14$ year old British adolescent females

21 (Jackson et al. 2013). Higher levels of peer acceptance have also been observed to

22 mitigate the negative impact of early maturation upon self-reported physical activity in

23 another sample ( $\mathrm{n}=342$; 7-9 year old) of adolescent British girls (Pindus et al. 2014). The 
1 results of these studies should, however, be interpreted with caution as the majority have

2 involved fairly small samples and self-reported measures of physical activity, which are

3 subject to biases associated with social desirability and recall (Shephard 2003).

4 The aim of the present paper was to test the mediational effects of body attractiveness and 5 physical self-worth on the relationship between biological maturity and accelerometer 6 assessed MVPA in a large multi-ethnic sample of girls from the Midlands area in the UK $7 \quad(11-14$ years $)$

\section{Method}

\section{Participants}

11 Cross sectional data for analyses were obtained from the baseline assessment of a cluster

12 randomized controlled trial (RCT) that aimed to evaluate the effectiveness of a school-

13 based physical activity intervention for girls. The protocol (Edwardson et al. 2015) and

14 results (Harrington et al. 2018) of the trial have been published previously. In brief, the

15 RCT included 20 secondary (high) schools in the Midlands area of the UK.

16 Approximately 90 girls, 11-14 year old, were invited at random to participate from each

17 school. After receiving Head Teacher consent, parents were asked to provide opt-out

18 consent (i.e., contact the school/researchers if they did not wish their child to take part)

19 and verbal assent was obtained from pupils. Ethical approval was obtained from the

20 University of Leicester College of Medicine and Biological Sciences (4667-mlh31-

21 diabetesresearchcentre).

23 Measures 
1 Participants completed a series of self-report questionnaires, including demographic

2 information and subscales from the Physical Self-Perception Profile (Whitehead 1995).

3 Standing height, weight and seated height were measured using standardized procedures.

4 Chronological age in decimals was determined by the difference between date of birth

5 and date of measurement.

6 Accelerometry

7 Participants were asked to wear the GENEActiv Original accelerometer (Activinsights

8 Ltd, Kimbolton, UK) continuously (i.e., 24 h/day) for seven days on their non-dominant

9 wrist. This device is a valid and reliable objective measure of physical activity in young

10 people (Schaefer et al. 2014). An in-depth description of the accelerometer processing

11 can be found here (Hansen et al. 2018 Apr 6). In brief, the devices were initialized to

12 collect data at $100 \mathrm{~Hz}$ and the data downloaded using GENEActiv PC software version

13 2.9. The GENEActiv.bin files were processed and acceleration averaged over 5 second

14 epochs using R-package GGIR version 1.2-2 (http://cran.r-project.org) (Migueles, J.H.,

15 Rowlands, A.V., Huber, F., Sabia, S., van Hees 2019). Detection of non-wear followed

16 the protocol published by Van Hees (van Hees et al. 2013) and was estimated on the basis

17 of the SD and value range of each axis, calculated for 60-min windows with a 15-min

18 sliding window. The window is classified as non-wear if, for at least two of the three

19 axes, the standard deviation (SD) is less than $13 \mathrm{mg}$ or the value range is less than $50 \mathrm{mg}$.

20 Monitored days were classified as invalid and excluded if wear time was less than $16 \mathrm{~h}$

21 (Edwardson et al. 2015). Physical activity was expressed as total time accumulated in

22 MVPA, defined as time accumulated above an acceleration of $200 \mathrm{mg}$ (Hildebrand et al.

23 2014) 


\section{$2 \quad$ Predicting Biological Maturity}

3 Stature (i.e., standing height), sitting height, leg length (stature minus sitting height), age,

4 and their interactions were used to predict how many years a girl is from APHV (Mirwald

5 et al. 2002), an indicator of biological maturity. This method assumes that a girl who

6 experiences APHV at an earlier age is more advanced in their biological maturity than a

7 girl who achieves it at a later age.

\section{$9 \quad$ Perceptions of the Physical Self}

10 Two adapted subscales from the physical self-perception profile (PSPP; Whitehead 1995)

11 were used to assess perceived physical self-worth and body attractiveness. These

12 subscales were completed by participants in the Girls Active Study as part of a larger

13 battery of self-report questionnaires. To limit the number of items participants were

14 required to complete, the other subscales from the PSPP were not included. Both

15 subscales required participants to indicate their agreement with a series of 6 items on a

16 five-point Likert scale, with responses ranging from 'strongly disagree' to 'strongly

17 agree'. Theoretically, physical self-worth is a higher-order construct, with body

18 attractiveness acting as lower-order construct, contributing to physical self-worth. Both

19 subscales demonstrated good levels of internal consistency in the current sample

20 (Physical self-worth: Cronbach's alpha =.85; Body attractiveness Cronbach's alpha =.83).

\section{Statistical Analyses}


1 Descriptive statistics were calculated for anthropometric variables, predicted APHV,

2 body attractiveness, physical self-worth, accelerometer wear time (days), and MVPA.

3 Pearson product moment correlations (one-tailed) were then calculated to investigate

4 relations among the primary variables of interest. Finally, structural equation modeling

5 (SEM), utilizing maximum likelihood estimation and bootstrapping procedures, was used

6 to assess the model fit. To assess the adequacy of model fit, a 2-index presentation

7 strategy advanced by $\mathrm{Hu}$ and Bentler (Hu and Bentler 1999) was employed. This strategy

8 uses the Standardized Root Mean Square Residual (SRMR) and incremental or absolute

9 indexes of fit (e.g., Comparative Fit Index -CFI). An SRMR value at or below .08 is

10 indicative of a well-specified model whereas CFI values of .90 and .95 represented

11 acceptable and excellent model fit, respectively (Hu and Bentler 1999). In line with

12 recommended practice (McKinnon et al. 2016), the mediated effects were explored by

13 examining the $90 \%$ upper and lower limits of bootstrap-generated bias-corrected

14 confidence intervals (BBC CI) of indirect effects.

\section{Descriptive Statistics}

18 Of the baseline sample, 691 (39\%) were excluded because there were missing

19 data on physical self-worth/body attractiveness, accelerometry and/or predicted APHV.

20 Therefore, the final sample retained for the analysis was 1062 (Mean age $=12.8$ years,

$21 \mathrm{SD}=0.8$ years; range $=11-14$ years $)$. There were no significant differences between

22 those excluded and those included in the analyses in any of the descriptive variables.

23 Approximately $79 \%$ of the sample described themselves as white, $9 \%$ as Asian, $4 \%$ as

24 Black, 5\% as mixed race, and 3\% as other. Descriptive statistics for chronological age, 
1 ethnicity, body size, and predicted APHV, body attractiveness, physical self-worth, and

2 physical activity are summarized by chronological age group in Table 1 . In all age

3 groups, the mean value for APHV was slightly greater than the expected mean of 12.1

4 years in European females. (Malina, Robert M. Bouchard, Claude. Bar-Or 2004)

5 Compared to UK-WHO reference values (Freeman et al. 1995), mean heights fell

6 between the $50^{\text {th }}$ and 75 th centiles for age at 12 and 13 years of age, and approximated

7 the $50^{\text {th }}$ centile at 11 and 14 years of age. Mean values for weight were between the $50^{\text {th }}$

8 and $75^{\text {th }}$ centiles at age 11 and 12 and approximated the $75^{\text {th }}$ centile at 13 years and 14

9 years. Mean values for BMI lay between the $50^{\text {th }}$ and $75^{\text {th }}$ centiles from 11 to 13 years of

10 age and approximated the $75^{\text {th }}$ centile at 14 years of age.

[Table 1 near here]

\section{Correlations}

15 Pearson product moment correlations between measures of maturation, body size,

16 body attractiveness, physical self-worth, and physical activity are presented in Table 2.

17 As expected, predicted APHV was positively associated with higher perceptions of

18 attractiveness, physical self-worth, and minutes engaged in MVPA. Similarly, minutes

19 spent in MVPA was positively associated with higher perceptions of attractiveness and

20 physical self-worth. 
MATURATION, PHYSICAL SELF, PHYSICAL ACTIVITY

\section{$1 \quad$ The Mediated Effects Model}

Structural equation modelling using maximum likelihood estimation was used to

3 test the fit of the hypothesized model (see Figure 1). Inspection of the Mardia's

4 Coefficient value $(41.20, p<.001)$ indicated that the data departed from multivariate

5 normality. Accordingly, the SEM analysis was performed using the bootstrapping

6 procedure with 5000 bootstrap replication samples to provide more accurate assessments

7 of the parameter estimates' stability (Bryne 2001).

[Figure 1 near here]

11 The model fit indices demonstrated a good level of fit between the proposed

12 model and the data $(\mathrm{SRMR}=.06 ; \mathrm{CFI}=.89)$. Modification indices $(>10.0)$ and

13 standardized residuals of the covariance matrix $(\mathrm{CR}>2.58)$ were examined to identify

14 potential areas of model misspecification. Only changes that were theoretically and

15 empirically substantive and resulted in statistically significant improvement in model fit

16 were considered. The standardised residuals did not identify any areas of model

17 misspecification. The modification indices did, however, suggest that model fit could be

18 improved by correlating a number of the error variances associated with psychometric

19 items that shared a common subscale $(\mathrm{CFI}=.93$; SRMR $=.05)$. As these modifications

20 did not result in any substantive changes to magnitude and direction of the path

21 coefficients, and for easier comparison and evaluation of the model in future studies, we

22 are presenting the results for the original non-modified model. Versions of the model

23 controlling for ethnicity and deprivation were also conducted, however, as these variables 
1 did not impact model fit and/or direction or statistical significance of any of the direct and

2 indirect parameter estimates they were not included in this paper.

3 The standardized Beta coefficients, standard errors, and squared multiple

4 correlations associated with the original hypothesized model are presented in Figure 2.

5 APHV was positively associated with perceived body attractiveness $(\beta=.25, \mathrm{p}<.001)$.

6 Perceived body attractiveness positively predicted physical self-worth $(\beta=.91, \mathrm{p}<.001)$

7 which, in turn, positively predicted greater MVPA $(\beta=.22, \mathrm{p}<.001)$. Predicted APHV was

8 found to positively and indirectly predict both physical self-worth $(\beta=.23(90 \% \mathrm{CI} \pm$

$9 \quad .05), \mathrm{p}<.001)$ and MVPA $(\beta=.05(90 \% \mathrm{CI} \pm .02, \mathrm{p}<.001), \mathrm{p}<.001)$. An indirect and

10 positive association between perceived body attractiveness and MVPA was also observed

$11 \quad(\beta=.20(90 \% \mathrm{CI} \pm .05), \mathrm{p}<.001)$.

12 Baron and Kenny's protocol for testing meditational hypotheses (Baron and

13 Kenny 1986), adapted for SEM (Shrout and Bolger 2002), was used to test for mediation

14 of the indirect effect between APHV and MVPA. Bootstrap-generated bias-corrected

15 confidence intervals were employed to estimate the standardized path coefficients

16 representing the direct effect, with and without the mediating variables. Without the

17 mediating variables the direct path coefficient between APHV and MVPA was

18 statistically significant $(\beta=.11(\mathrm{BBC} 90 \% \mathrm{CI}=.06, .16), \mathrm{p}<.001)$. When the mediating

19 variables were included within the model the path coefficient representing the direct

20 effect between APHV and MVPA was attenuated but remained statistically significant ( $\beta$

$21=.06(\mathrm{BBC} 90 \% \mathrm{CI}=.01, .11), \mathrm{p}=.04)$. This result indicates that the association between

22 biological maturation (i.e. APHV) and MVPA is only partially mediated and that a 
1 significant proportion of the variance shared between the two constructs remains

2 unexplained.

[Figure 2 near here]

\section{Discussion}

7 The results of the current investigation provide partial support for the hypothesized

8 mediated effects model and supported the contention that advanced (i.e. early) maturation

9 in adolescent girls is associated with lower MVPA. Consistent with previous literature

10 (Jackson et al. 2013), later maturation was found to be associated with higher perceptions

11 of body attractiveness and physical self-worth, which, in turn, predicted greater MVPA.

12 The nature and directions of the path coefficients observed in the hypothesized model

13 were also consistent with those described in previous studies examining the mediated

14 effects of maturation upon physical activity in adolescent girls (Cumming, Standage, et

15 al. 2011). It should be noted, however, that the direct association between maturation and

16 MVPA remained statistically significant when the mediating variables were included in

17 the model. This suggests that perceptions of the physical self may account for some but

18 not all of the variation between maturation and MVPA. The unexplained variance may

19 result from a direct effect of maturation on MVPA or may be a product of variables that

20 are not accounted for in the hypothesized model.

21 The observed associations among maturation, body attractiveness, and physical

22 self-worth are worthy of further discussion. Girls who mature in advance of their peers

23 appear to view themselves as less physically attractive and, as a consequence, report 
1 lower physical self-worth. Physical appearance is considered an important source of self-

2 worth and esteem in adolescent girls (Harter 2000). A study of over 50 US adolescent

3 girls and boys suggested that body attractiveness was the most common subject of

4 conversation for girls during adolescence and was deemed to be of greater importance

5 than one's success in education and sports (Martin 1996). This observation that

6 perceptions of the physical self are lower in girls who are more advanced in maturation

7 may result from the maturity associated changes in size, shape and physique that

8 accompany puberty. Many of these changes run counter to Western ideals pertaining to

9 attractiveness in females (i.e., preference towards a more linear, lean physique) (Paxton et

10 al. 2005). Girls who mature in advance of their peers will generally experience a more

11 intense pubertal growth spurt. (Malina, Robert M. Bouchard, Claude. Bar-Or 2004) Early

12 maturing girls will also experience these changes at an age when they may not possess

13 the cognitive or emotional skills to respond and/or adapt effectively (Pringle et al. 2017).

14 The results of the current study are useful from an applied perspective as they

15 identify a potential mechanism through which maturity associated declines in physical

16 activity might be countered. Physical self-worth represents the individual's beliefs,

17 knowledge, values and perceptions pertaining to the body (Fox and Lindwall) and can be

18 modified through personalized feedback education, challenging perceptions and re-

19 evaluation (Manning 2007). A lack of knowledge pertaining to the physiological

20 processes of normal growth and maturation has been documented as a source of anxiety

21 in adolescent girls (Martin 1996). Psychoeducational interventions designed to inform

22 adolescents on the subject of puberty and encourage them to accept it as a normal and

23 attractive part of becoming an adult, may be of value in terms of helping students adapt to 
1 this change more effectively. Such intervention should combine education with the

2 personal reorganization and reinterpretation of body image and related self-perceptions.

3 The intervention should also encourage students to challenge existing stereotypes

4 pertaining to the body and might borrow principles and/or strategies from existing

5 Cognitive Behavioral Therapy interventions (Cash, T. F. 1997). While there is much

6 evidence to support the effectiveness of body-image CBT programs in college aged and

7 adult samples, the efficacy of such interventions in adolescents is less clear. Further

8 research on the design, implementation and efficacy of such strategies, and how they

9 might be incorporated into physical activity programmes for adolescent girls is

10 warranted. Researchers investigating physical activity correlates/determinants and bio-

11 social theories of physical activity/behavior change of adolescents should also consider

12 including timing of biological maturity. Furthermore, investigate whether effect of

13 physical activity interventions varies by biological maturity status, in addition to other

14 important intrapersonal characteristics such as gender, ethnicity and socioeconomic status

15 may also be warranted.

16 Strengths of the study include a large multi-ethnic female sample and device measured

17 physical activity, with high compliance. However, these findings may not transfer to

18 adolescent boys. Although boys progress through a comparable process, the average age

19 and nature of the physical changes that occur are markedly different (de Guzman and

20 Nishina 2014). For example, early maturity in males is associated with enhanced lean

21 mass resulting in physiological (e.g. strength and power) advantages. Thus, adolescent

22 boys' perceptions of early maturity are likely to be quite different to adolescent girls.

23 However, to date there is a paucity of research investigating this. Unfortunately, our 
1 sample size did not permit us the mediation models to be run by ethnic group (i.e. white

2 British and South Asian). The importance of body attractiveness to overall self-worth and

3 what defines body attractiveness has been shown to vary with culture and social class

4 (Martin 1996). African American girls, for instance, tend to be more satisfied with their

5 bodies than white girls (de Guzman and Nishina 2014). To the authors' knowledge there

6 is a paucity of data on how South Asian girls perceive the physical changes that occur

7 during adolescence. However, there is literature to suggest that South Asian girls may be

8 at more risk for developing a negative body image compared with white British (Bakhshi

9 2011), but more research with larger samples of South Asian girls is needed. Due to the

10 cross-sectional nature of this study's design it is also not possible to comment on

11 causation. Longitudinal research is required, ideally beginning in late childhood and

12 tracking changes through to late adolescence in order to better understand how physical

13 self-perceptions may mediate relations between maturation and physical activity. It is

14 important to note limitations associated with the method for estimating maturation. The

15 reliability and accuracy of the maturity offset has been shown to vary relative to both age

16 and maturity timing, with errors being greatest in older and younger children, and

17 children who are especially advanced or delayed in maturation (Malina and Kozieł 2014).

18 Lastly, examining a broader range of self-perceptions (e.g. global self-worth, sport

19 competence, strength, physical condition) may have influenced results.

20 In conclusion, the results of the current study provide partial support for the

21 mediated effects of model of maturity associated variation in physical activity and the

22 hypothesis that advanced maturation in adolescent females is associated with less

23 involvement in MVPA. As a consequence, researchers interested in the study of physical 
1 activity, including the development of targeted interventions for adolescent girls, should

2 consider the measurement of maturation status to explore the potential contribution of

3 biological maturation, including interactions with psychosocial constructs. 
MATURATION, PHYSICAL SELF, PHYSICAL ACTIVITY

References

2 Bakhshi S. 2011. Women's body image and the role of culture: A review of the literature.

3 Eur J Psychol. 7(2):374-394. doi:10.5964/ejop.v7i2.135.

4 Baron RM, Kenny DA. 1986. The moderator-mediator variable distinction in social

5 psychological research: Conceptual, strategic, and statistical considerations. J Pers Soc

6 Psychol. 51(6):1173-1182. doi:10.1037/0022-3514.51.6.1173. [accessed 2019 Dec 11].

7 http://doi.apa.org/getdoi.cfm?doi=10.1037/0022-3514.51.6.1173.

8 Bryne B. 2001. Structural Equation Modeling with Amos: Basic Concepts, Applications,

9 and Programming. NJ, LEA,: Mahwah.

10 Cash, T. F. 1997. The Body-Image Workbook: An Eight Step Program for Learning to

11 Like Your Looks. New Harbinger, CA: Oakland.

12 Cumming SP, Gillison FB, Sherar LB. 2011. Biological maturation as a confounding

13 factor in the relation between chronological age and health-related quality of life in

14 adolescent females. Qual Life Res. 20(2). doi:10.1007/s11136-010-9743-0.

15 Cumming SP, Sherar LB, Pindus DM, Coelho-e-Silva MJ, Malina RM, Jardine PR. 2012.

16 A biocultural model of maturity-associated variance in adolescent physical activity. Int

17 Rev Sport Exerc Psychol. 5(1). doi:10.1080/1750984X.2011.630481.

18 Cumming SP, Sherar LB, Smart JE, Rodrigues AMM, Standage M, Gillison FB, Malina

19 RM. 2012. Physical Activity, Physical Self-Concept, and Health-Related Quality of Life

20 of Extreme Early and Late Maturing Adolescent Girls. J Early Adolesc. 32(2).

21 doi:10.1177/0272431610393250.

22 Cumming SP, Standage M, Loney T, Gammon C, Neville H, Sherar LB, Malina RM.

23 2011. The mediating role of physical self-concept on relations between biological

24 maturity status and physical activity in adolescent females. J Adolesc. 34(3).

25 doi:10.1016/j.adolescence.2010.06.006.

26 Edwardson CL, Harrington DM, Yates T, Bodicoat DH, Khunti K, Gorely T, Sherar LB,

27 Edwards RT, Wright C, Harrington K, et al. 2015. A cluster randomised controlled trial

28 to investigate the effectiveness and cost effectiveness of the "Girls Active" intervention:

29 A study protocol. BMC Public Health. 15(1). doi:10.1186/s12889-015-1886-z.

30 Ekelund U, Sherar L, Cooper A. 2012. Time spent sedentary and active and

31 cardiometabolic risk factors in children - In reply. JAMA - J Am Med Assoc. 307(19). 
1 Fox KR, Lindwall M. Self-esteem and self-perceptions in sport and exercise. In:

2 Routledge Companion to Sport and Exercise Psychology. Routledge. [accessed 2020 Jan

3 21]. https://www.taylorfrancis.com/books/9781315880198.

4 Freeman J V., Cole TJ, Chinn S, Jones PRM, White EM, Preece MA. 1995. Cross

5 sectional stature and weight reference curves for the UK, 1990. Arch Dis Child.

6 73(1):17-24. doi:10.1136/adc.73.1.17.

7 Guthold R, Stevens GA, Riley LM, Bull FC. 2019 Nov. Global trends in insufficient

8 physical activity among adolescents: a pooled analysis of 298 population-based surveys

9 with 1.6 million participants. Lancet Child Adolesc Heal. doi:10.1016/S2352-

10 4642(19)30323-2. [accessed 2019 Nov 26].

11 https://linkinghub.elsevier.com/retrieve/pii/S2352464219303232.

12 de Guzman NS, Nishina A. 2014. A longitudinal study of body dissatisfaction and

13 pubertal timing in an ethnically diverse adolescent sample. Body Image. 11(1):68-71.

14 doi:10.1016/j.bodyim.2013.11.001. [accessed 2020 Jan 21].

15 http://www.ncbi.nlm.nih.gov/pubmed/24331829.

16 Hansen BH, Anderssen SA, Andersen LB, Hildebrand M, Kolle E, Steene-Johannessen J,

17 Kriemler S, Page AS, Puder JJ, Reilly JJ, et al. 2018 Apr 6. Cross-Sectional Associations

18 of Reallocating Time Between Sedentary and Active Behaviours on Cardiometabolic

19 Risk Factors in Young People: An International Children's Accelerometry Database

20 (ICAD) Analysis. Sports Med. doi:10.1007/s40279-018-0909-1. [accessed 2018 Apr 29].

21 http://link.springer.com/10.1007/s40279-018-0909-1.

22 Harrington DM, Davies MJ, Bodicoat DH, Charles JM, Chudasama Y V., Gorely T,

23 Khunti K, Plekhanova T, Rowlands A V., Sherar LB, et al. 2018. Effectiveness of the

24 "Girls Active" school-based physical activity programme: A cluster randomised

25 controlled trial. Int J Behav Nutr Phys Act. 15(1):40. doi:10.1186/s12966-018-0664-6.

26 Harter S. 2000. Is Self-Esteem Only Skin-Deep? The Inextricable Link between Physical

27 Appearance and Self-Esteem. Reclaiming Child Youth. 9(3):133.

28 https://www.researchgate.net/publication/234657515_Is_Self-Esteem_Only_Skin-

29 Deep_The_Inextricable_Link_between_Physical_Appearance_and_Self-

30 Esteem/citation/download.

31 van Hees VT, Gorzelniak L, Dean León EC, Eder M, Pias M, Taherian S, Ekelund U, 
1 Renström F, Franks PW, Horsch A, et al. 2013. Separating Movement and Gravity

2 Components in an Acceleration Signal and Implications for the Assessment of Human

3 Daily Physical Activity. PLoS One. 8(4). doi:10.1371/journal.pone.0061691.

4 Hildebrand M, VAN Hees VT, Hansen BH, Ekelund U. 2014. Age group comparability

5 of raw accelerometer output from wrist- and hip-worn monitors. Med Sci Sports Exerc.

6 46(9):1816-24. doi:10.1249/MSS.0000000000000289. [accessed 2019 Dec 2].

7 http://www.ncbi.nlm.nih.gov/pubmed/24887173.

8 Hu LT, Bentler PM. 1999. Cutoff criteria for fit indexes in covariance structure analysis:

9 Conventional criteria versus new alternatives. Struct Equ Model. 6(1):1-55.

10 doi:10.1080/10705519909540118.

11 Jackson L, Cumming SP, Drenowatz C, Standage M, Sherar LB, Malina RM. 2013.

12 Biological maturation and physical activity in adolescent British females: The roles of

13 physical self-concept and perceived parental support. Psychol Sport Exerc. 14(4).

14 doi:10.1016/j.psychsport.2013.01.001.

15 Machado Rodrigues AM, Coelho E Silva MJ, Mota J, Cumming SP, Sherar LB, Neville

16 H, Malina RM. 2010. Confounding effect of biologic maturation on sex differences in

17 physical activity and sedentary behavior in adolescents. Pediatr Exerc Sci. 22(3).

18 Malina, Robert M. Bouchard, Claude. Bar-Or O. 2004. Growth, Maturation, and Physical

19 Activity. 22nd ed. Champagne, Ill: Human Kinetics. [accessed 2019 Dec 2].

20 https://books.google.co.uk/books/about/Growth_Maturation_and_Physical_Activity.html

$21 ? \mathrm{id}=\mathrm{VqF}$ FFsykj6EC\&redir_esc $=\mathrm{y}$.

22 Malina RM, Kozieł SM. 2014. Validation of maturity offset in a longitudinal sample of

23 Polish girls. J Sports Sci. 32(14):1374-1382. doi:10.1080/02640414.2014.889846.

24 Manning MA. 2007. Self-concept and self-esteem in adolescents. Student Serv. 2:11-15.

25 Martin KA. 1996. Puberty, Sexuality, and the Self: Girls and Boys at Adolescence. New

26 York: Routledge.

27 McKinnon B, Gariépy G, Sentenac M, Elgar FJ. 2016. Adolescent suicidal behaviours in

2832 low- and middle-income countries. Bull World Health Organ. 94(5):340-350F.

29 doi:10.2471/BLT.15.163295. [accessed 2019 Jul 5].

30 http://www.who.int/entity/bulletin/volumes/94/5/15-163295.pdf.

31 Migueles, J.H., Rowlands, A.V., Huber, F., Sabia, S., van Hees V. 2019. GGIR: A 
1 Research Community-Driven Open Source R Package for Generating Physical Activity

2 and Sleep Outcomes From Multi-Day Raw Accelerometer Data in: Journal for the

3 Measurement of Physical Behaviour Volume. J Meas Phys Behav. 2(3). [accessed 2020

4 Mar 6]. https://journals.humankinetics.com/view/journals/jmpb/2/3/article-p188.xml.

5 Mirwald RL, Baxter-Jones ADG, Bailey DA, Beunen GP. 2002. An assessment of

6 maturity from anthropometric measurements. Med Sci Sports Exerc. 34(4):689-694.

7 doi:10.1249/00005768-200204000-00020.

8 Paxton SJ, Norris M, Wertheim EH, Durkin SJ, Anderson J. 2005. Body dissatisfaction,

9 dating, and importance of thinness to attractiveness in adolescent girls. Sex Roles. 53(9-

10 10):663-675. doi:10.1007/s11199-005-7732-5.

11 Pindus DM, Cumming SP, Sherar LB, Gammon C, Coelho e Silva M, Malina RM. 2014.

12 Maturity-Associated Variation in Physical Activity and Health-Related Quality of Life in

13 British Adolescent Girls: Moderating Effects of Peer Acceptance. Int J Behav Med.

14 21(5). doi:10.1007/s12529-013-9344-8.

15 Pringle J, Mills KL, McAteer J, Jepson R, Hogg E, Anand N, Blakemore SJ. 2017. The

16 physiology of adolescent sexual behaviour: A systematic review. Cogent Soc Sci. 3(1).

17 doi:10.1080/23311886.2017.1368858.

18 Schaefer CA, Nigg CR, Hill JO, Brink LA, Browning RC. 2014. Establishing and

19 evaluating wrist cutpoints for the GENEActiv accelerometer in youth. Med Sci Sports

20 Exerc. 46(4):826-33. doi:10.1249/MSS.0000000000000150. [accessed 2019 Dec 2].

21 http://www.ncbi.nlm.nih.gov/pubmed/24121241.

22 Shephard RJ. 2003. Limits to the measurement of habitual physical activity by

23 questionnaires. Br J Sports Med. 37(3):197-206. doi:10.1136/bjsm.37.3.197.

24 Sherar LB, Cumming SP, Eisenmann JC, Baxter-Jones ADG, Malina RM. 2010.

25 Adolescent biological maturity and physical activity: Biology meets behavior. Pediatr

26 Exerc Sci. 22(3). doi:10.1123/pes.22.3.332.

27 Sherar LB, Esliger DW, Baxter-Jones ADG, Tremblay MS. 2007. Age and gender

28 differences in youth physical activity: Does physical maturity matter? Med Sci Sports

29 Exerc. 39(5). doi:10.1249/mss.0b013e3180335c3c.

30 Shrout PE, Bolger N. 2002. Mediation in experimental and nonexperimental studies: New

31 procedures and recommendations. Psychol Methods. 7(4):422-445. doi:10.1037/1082- 
$1989 X .7 .4 .422$.

2 Whitehead J. 1995. A study of children's physical self-perceptions using an adapted

3 physical self-perception profile questionnaire. Pediatr Exerc Sci. 7:132-51.

6 Funding Details: The study which this analysis is based on was funded by the National

7 Institute for Health Research (NIHR), Public Health Research Programme [13/90/30]

8 Professors Davies and Khunti are NIHR Senior Investigators. University of Leicester

9 authors are supported by the NIHR Leicester-Loughborough Biomedical Research Unit

10 (2012-2017), the NIHR Leicester Biomedical Research Centre (2017-2022) and the

11 Collaboration for Leadership in Applied Health Research and Care (CLAHRC) East

12 Midlands. The Girls Active study was undertaken in collaboration with the Leicester

13 Clinical Trials Unit a UKCRC-registered clinical trials unit in receipt of NIHR CTU

14 support funding and the Youth Sport Trust. The aforementioned funders had no

15 involvement in the TSC, the data analysis, data interpretation, data collection, or writing

16 of this manuscript. The views expressed are those of the authors and not necessarily those

17 of the NHS, the NIHR or the Department of Health and Social Care.

19 Conflict of Interest: No potential conflict of interest was reported by the authors. 
1 Table 1. Descriptive statistics for measures of maturation, body size, physical self-

2 perceptions, accelerometer wear time, and physical activity in British girls aged 11 to

314 years $(\mathrm{N}=1062)$.

4

\begin{tabular}{lcccc}
\hline & $\begin{array}{c}11 \text { years } \\
\mathrm{n}=194\end{array}$ & $\begin{array}{c}12 \text { years } \\
\mathrm{n}=403\end{array}$ & $\begin{array}{c}13 \text { years } \\
\mathrm{n}=354\end{array}$ & $\begin{array}{c}14 \text { years } \\
\mathrm{n}=111\end{array}$ \\
& $11.8(.1)$ & $12.5(.3)$ & $13.4(.3)$ & $14.3(.2)$ \\
\hline Chronological age (years) & $12.5(.7)$ & $12.6(.8)$ & $12.5(.8)$ & $12.7(.8)$ \\
Predicted age at PHV & & & & \\
(years) & $-.7(.8)$ & $-.1(.8)$ & $.9(.9)$ & $1.6(.9)$ \\
Years from APHV (years) & $150.9(7.2)$ & $155.1(7.6)$ & $159.5(6.9)$ & $161.1(6.6)$ \\
Height (cm) & $43.3(10.1)$ & $46.4(10.8)$ & $53.0(12.8)$ & $55.9(13.2)$ \\
Weight (kg) & $18.8(3.4)$ & $19.1(3.5)$ & $20.7(4.3)$ & $21.5(4.6)$ \\
BMI (kg/m $\left.{ }^{2}\right)$ & $3.5(.9)$ & $3.4(.9)$ & $3.1(.8)$ & $2.9(.9)$ \\
Physical self-worth & $2.9(.9)$ & $2.9(.9)$ & $2.6(.8)$ & $2.6(.9)$ \\
Body attractiveness & $6.9(.1)$ & $6.9(.1)$ & $6.9(.1)$ & $6.9(.1)$ \\
Accel wear time (days) & $39.5(8.4)$ & $37.2(8.6)$ & $34.5(7.8)$ & $33.2(7.7)$ \\
MVPA (mins/day) & & &
\end{tabular}

5

$6 \quad \mathrm{MVPA}=$ moderate - to-vigorous physical activity; $\mathrm{APHV}=$ age at

7 peak height velocity; BMI= Body Mass Index 
Table 2. Pearson product moment correlations (one-tailed) between measures of maturation, body size, physical self-perceptions, and physical activity in a sample of UK girls aged 11 to 14 years $(\mathrm{N}=1062)$.

\begin{tabular}{|c|c|c|c|c|c|c|}
\hline & 1 & 2 & 3 & 4 & 5 & 6 \\
\hline \multicolumn{7}{|l|}{ 1. Predicted APHV } \\
\hline 2. Height & $-.63 * * *$ & & & & & \\
\hline 3. Weight & $-.87 * * *$ & $.64 * * *$ & & & & \\
\hline 4. BMI & $-.78 * * *$ & $.32 * * *$ & $.93 * * *$ & & & \\
\hline 5. Body attractiveness & $.25 * * *$ & $-.11 * * *$ & $-.35 * * *$ & $-.38 * * *$ & & \\
\hline 6. Physical self-worth & $.24 * * *$ & $-.16^{* * *}$ & $-.34 * * *$ & $-.35 * * *$ & $.78 * * *$ & \\
\hline 7. MVPA & $.11 * * *$ & $-.29 * * *$ & $-.17 * * *$ & $-.08 *$ & $.16^{* * *}$ & $.22 * * *$ \\
\hline
\end{tabular}


1 Figure 1. Hypothesized mediated effects model describing relations among biological

2 maturity, perceived body attractiveness and physical self-worth, and MVPA in adolescent 3 females.

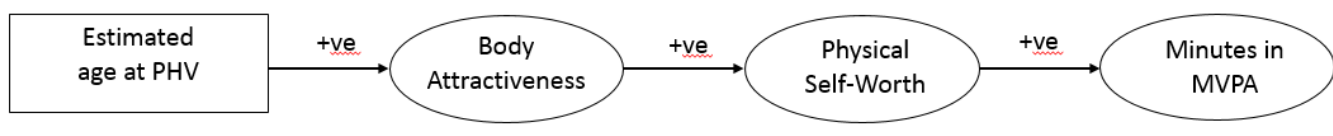


1

2 Figure 2. Mediated effects model describing relations among biological maturity,

3 perceived body attractiveness and physical self-worth, and MVPA in adolescent females.

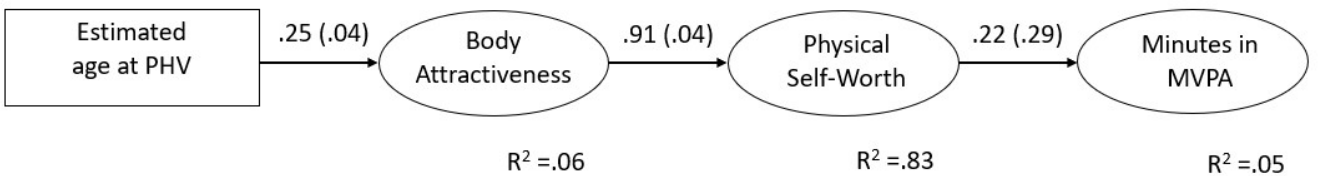

5

$6 \quad$ Note. All solid line parameters are significant $(\mathrm{p}<.05)$. Standardized Beta

7 coefficients are presented for each parameter with standard errors in parentheses. Squared

8 multiple correlations included for all endogenous factors. Indirect effects were observed

9 between APHV and physical self-worth $(\beta=.28(\mathrm{BBC} 90 \% \mathrm{CI}=.39,17), \mathrm{P}<.001)$,

10 APHV and MVPA $(\beta=.14(\mathrm{BBC} 90 \% \mathrm{CI}=22, .08), \mathrm{P}<.001)$, and perceived body

11 attractiveness and MVPA $(\beta=.17(\mathrm{BBC} 90 \% \mathrm{CI}=25, .10), \mathrm{P}<.001)$. Factors indicators

12 are not included in the model for the purpose of making the presentation less complex.

13

14 